\title{
Laponite as carrier for controlled in vitro delivery of dexamethasone in vitreous humor models
}

José M. Fraile, ${ }^{a} *$ Elena Garcia-Martin, ${ }^{b, c}$ Cristina Gil, ${ }^{a}$ José A. Mayoral, ${ }^{a}{ }^{*}$ Luis E. Pablo, ${ }^{b, c}$ Vicente Polo, ${ }^{b, c}$ Esther Prieto, ${ }^{b, c}$ Eugenio Vispe ${ }^{d}$

a Instituto de Síntesis Química y Catálisis Homogénea (ISQCH), Facultad de Ciencias, Universidad de Zaragoza-CSIC, C/ Pedro Cerbuna 12, E-50009 Zaragoza, Spain.

${ }^{\mathrm{b}}$ Departamento de Oftalmología, Hospital Universitario Miguel Servet, C/ Padre Arrupe, Edificio Consultas Externas, E-50009 Zaragoza, Spain.

c Instituto de Investigación Sanitaria Aragón (IIS-Aragón), Universidad de Zaragoza, Facultad de Medicina, C/ Domingo Miral s/n, E-50009 Zaragoza, Spain.

${ }^{d}$ Laboratorio de Cromatografía y Espectroscopia, Instituto de Síntesis Química y Catálisis Homogénea (ISQCH), Facultad de Ciencias, Universidad de Zaragoza-CSIC, C/ Pedro Cerbuna 12, E-50009 Zaragoza, Spain.

*Corresponding Authors. E-mail: jmfraile@unizar.es; mayoral@unizar.es

KEYWORDS: dexamethasone, controlled delivery, laponite, corticosteroids, vitreous humor

ABSTRACT. Laponite clay is able to retain dexamethasone by simple physisorption, presumably accomplished by hydrogen bonding formation and/or complexation with sodium counterions, as shown by solid state NMR. The physisorption can be somehow modulated by changing the solvent in the adsorption process. This simple system is able to deliver dexamethasone in a controlled manner to solutions used as models for vitreous humor. The proved biocompatibility of laponite as well as its transparency in the gel state, together with the simplicity of the preparation method, make this system suitable for future in vivo tests of ophthalmic treatment. 


\section{Introduction}

Among the treatments for posterior segment ocular diseases, intravitreal injection is considered an effective approach, as it delivers the therapeutic agent directly into the eye $[1,2]$. In spite of some advantages with respect to systemic administration, such as lower side effects, intravitreal injection presents some potential risks associated to the need for repeated injections [3]. In the last years, alternative drug delivery systems are being developed to overcome this kind of limitations by reducing the frequency of injections, and hence the potential complications [4-6]. For example, dexamethasone (DEX) has been released from biodegradable subconjunctival implants of a poly(ortho ester) [7], from intravitreal implants

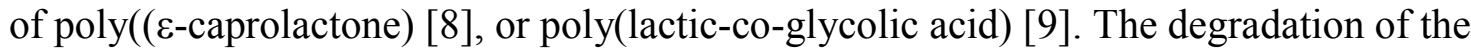
polymeric matrix produces the slow release of DEX, but this methodology requires a surgical intervention to implant the material $[8,10]$.

Very recently an intravitreal injectable implant has been described using DEX encapsulated into a thermogel matrix of poly(lactic acid-co-glycolic acid)-poly(ethylene glycol)poly(lactic acid-co-glycolic acid) triblock copolymer, able to lengthen the ocular retention time of DEX from several hours to more than one week [11]. In this case the matrix was prepared by copolymerization of lactic acid and glycolic acid, using poly(ethylene glycol) as initiator. Other hydrogels based on poly(ethylene glycol) and poly(valerolactone) [12] or hyaluronic acid $[13,14]$ have been also used for DEX release, but not in ophthalmic applications.

Clay minerals play different roles in health care formulations $[15,16]$, mainly for topical and gastrointestinal diseases, but only recently they have been envisaged as drug delivery modulators [17-19]. Natural clays show a certain degree of variability in their properties, such as chemical composition, particle size, and color. Hence, it is very difficult to envisage their use in intravitreal applications, and only in one case they have been used as components of polyurethane composites for implants releasing dexamethasone acetate upon degradation of the polymer [20]. However, synthetic clays are prepared with reproducible composition and textural properties. As an example, laponite (LAP) is a white synthetic smectite clay, a layered hydrous magnesium silicate with an empirical formula of $\mathrm{Na}_{0.7}\left[\left(\mathrm{Si}_{8} \mathrm{Mg}_{5.5} \mathrm{Li}_{0.3}\right) \mathrm{O}_{20}(\mathrm{OH})_{4}\right]$ that forms transparent colloidal dispersions in water [21]. As sodium cations are exchangeable, this property has been used to immobilize cationic drugs, usually molecules with protonated amino groups, that can be released in aqueous saline 
solutions. This is the case of itraconazole [22] (anti-fungal drug), donepezil [23] (Alzheimer's disease), tetracycline [24] (antimicrobial agent), and doxorubicin [25] (antitumoral). In vivo studies with the doxorubicin-laponite system have shown a high antitumor efficacy of the system with very low toxicity [26], whereas the laponite disks can be modified with folic acid for targeted drug delivery [27].

Here, we describe the first report of immobilization a non-ionic drug, dexamethasone, on laponite clay, the nature of the drug-clay interaction and the in vitro behavior of the material in controlled delivery.

\section{Materials and methods}

\subsection{Materials}

Dexamethasone (DEX) was from Aldrich. Laponite (LAP) was from Rockwood Additives. Ethanol, acetone and acetonitrile were HPLC grade from Scharlab. $\mathrm{CD}_{2} \mathrm{Cl}_{2}, \mathrm{CD}_{3} \mathrm{OD}$, acetone$d_{6}$ and dmso- $d_{6}$ were from Aldrich. Sodium hyaluronate $3 \%$ solution (Healon ${ }^{\circledR}$ EndoCoat OVD) was from Abbott Medical Optics. Saline solution $(9 \mathrm{mg} / \mathrm{mL} \mathrm{NaCl})$ was from Fresnius Kabi España.

\subsection{Preparation and characterization of $D E X / L A P$}

The required amount of DEX (20, 10 or $5 \mathrm{mg})$ was dissolved in ethanol or acetone $(10 \mathrm{~mL})$ and LAP (100 mg) was added to this solution. The slurry was stirred at room temperature for $1 \mathrm{~h}$ and then the solvent was removed in a rotary evaporator. In that way, the whole amount of DEX was deposited on the laponite support. The solid was dried at room temperature overnight under vacuum over $\mathrm{P}_{2} \mathrm{O}_{5}$.

DEX contents were assessed (less than $0.5 \%$ error) by carbon analysis of the dried powders in a Perkin-Elmer EA-2400 Series II elemental analyzer. Extraction of the samples with acetonitrile for $1 \mathrm{~h}$ led to the complete release of DEX to solution, as determined by HPLC (see below). FT-IR spectra (250 scans) of KBr pellets were registered using a ThermoNicolet Nexus 5700 spectrometer in the range from 4000 to $600 \mathrm{~cm}^{-1}$ at a resolution of 4 $\mathrm{cm}^{-1} \cdot{ }^{13} \mathrm{C}$ CP-MAS NMR (cross-polarization magic angle spinning nuclear magnetic resonance) spectra were recorded in a Bruker Avance III WB400 spectrometer with $4 \mathrm{~mm}$ zirconia rotors spun at magic angle in $\mathrm{N}_{2}$ at $10 \mathrm{kHz}$. Spectra (1000-25000 scans depending on 
the sample) were measured using a ${ }^{1} \mathrm{H} \pi / 2$ pulse length of $3 \mu \mathrm{s}$, with a contact time (ramp) of $3 \mathrm{~ms}$, spinal64 proton decoupling sequence of $5.3 \mu$ s pulse length and recycle delay of $7 \mathrm{~s}$. Dipolar dephasing (DD) spectra were recorded under the same conditions with a dephasing delay of $50 \mu \mathrm{s}$ and a refocusing ${ }^{13} \mathrm{C} \pi$ pulse of $8 \mu \mathrm{s}$. Solution NMR spectra were recorded in a Bruker Avance 400 spectrometer. Step scanned (1s/step) X-ray diffraction patterns were collected in the Servicio de Difracción de Rayos X y Análisis por Fluorescencia del Servicio General de Apoyo a la Investigación de la Universidad de Zaragoza at room temperature from $3^{\circ}$ in $2 \theta$ up to $35^{\circ}$, using a D-Max 2500 Rigaku system with a rotating anode. CuK $\alpha$ radiation with a wavelength of $1.54 \AA$ at $40 \mathrm{kV}$ and $80 \mathrm{~mA}$ was used.

\subsection{In vitro DEX release studies}

Release was studied in two extraction media, saline solution and a model of vitreous humor made up of $0.5 \%$ sodium hyaluronate in saline solution. The release tests were performed by dispersing DEX/LAP $(20 \mathrm{mg})$ in the extraction medium $(2 \mathrm{~mL}$ of saline or $2 \mathrm{~g}$ of the vitreous model) under stirring at $120 \mathrm{rpm}$ at room temperature. After $24 \mathrm{~h}$ the dispersion was centrifuged at $14000 \mathrm{rpm}$ for $20 \mathrm{~min}$. The liquid phase was removed and analyzed, while the solid was re-dispersed in fresh extraction medium for a new cycle.

The analysis of DEX was carried out by HPLC in a Waters 2695 system equipped with a Phenomenex Kinetex C18 column $(75 \mathrm{~mm} \times 4.6 \mathrm{~mm} \times 2.6 \mu \mathrm{m})$ coupled to a Waters 2995 PDA detector. The mobile phase was acetonitrile/water (35:65 v/v) pumped at a flow rate of $0.2 \mathrm{~mL} / \mathrm{min}$ at $35^{\circ} \mathrm{C}$. Detection at $250 \mathrm{~nm}$ was used. The amount of DEX was calculated by a seven point calibration curve against 6 - $\alpha$-methylprednisolone (internal standard). Aliquots of the extraction medium $(200 \mu \mathrm{L})$ were mixed with acetonitrile $(2 \mathrm{~mL})$ and $100 \mu \mathrm{L}$ of a solution of standard (20 ppm in acetonitrile). The solution was then vortexed for $1 \mathrm{~min}$ and sonicated for $5 \mathrm{~min}$ to ensure the complete mixing, and finally centrifuged at $3000 \mathrm{rpm}$ for 5 min. The supernatant was collected and evaporated under vacuum, then redissolved in 200 $\mu \mathrm{L}$ of acetonitrile, filtered through a $0.22 \mu \mathrm{m}$ PTFE syringe filter and analyzed by HPLC. The presence of laponite in the supernatant was not detected in any case. 


\section{Results and discussion}

\subsection{Characterization of adsorbed DEX}

In contrast with other drugs immobilized on LAP [22-25], DEX is not cationic and it cannot interact with the solid surface through electrostatic forces. Thus, it was deposited on LAP by suspending the clay in an ethanol solution of the drug, with three different DEX/LAP weight ratios, 1:20, 1:10 and 1:5, labeled as low, medium and high, as the optimal situation would be the use of the minimum amount of support for intravitreal injections. The solids were obtained by simply evaporating the solvent under reduced pressure.

As the immobilization of DEX on LAP cannot be due to electrostatic interactions through the exchange of sodium cations, the distribution of DEX on the surface is not easily predictable. X-ray diffraction was used to determine whether the dexamethasone molecules were able to enter the interlayer space of laponite, leading to an increase in the basal spacing of the clay. As can be seen in Figure 1A, the powder patterns are poorly oriented, and the 001 line is not clearly defined and it appears as a shoulder. In DEX(high)/LAP, the sharp diffraction lines corresponding to pure orthorhombic DEX (JCPDS database) are observable, indicating the presence of DEX crystallites. This pattern is different from the one registered with the aspurchased DEX (Figure 1). This difference might be explained by the process of solution and crystallization on the laponite surface used in the preparation of the DEX/LAP samples. Oriented samples of LAP can be obtained by addition of some drops of a solvent, for example acetone. In such case (Figure 1B), the basal spacing of laponite (14.3 $\AA$ ) is in good agreement with the layer thickness $(9.3 \AA)$ [21] and the interlayer space (5.0 $\AA$ ) is compatible with the presence of partially hydrated sodium cations (diameter of the fully hydrated cation $=7.2 \AA[28]$ ) in the interlayer space. However, the treatment of DEX/LAP with acetone produces the redistribution of DEX, given its solubility in acetone, as shown by the presence of DEX particles even at low content. In that case, a basal spacing of $21.5 \AA$ is observable, which can be attributed to the presence of DEX crystallites in the interlayer space. Although the presence of intercalated DEX molecules between the clay layers is not directly detected, it can be inferred from this result. 

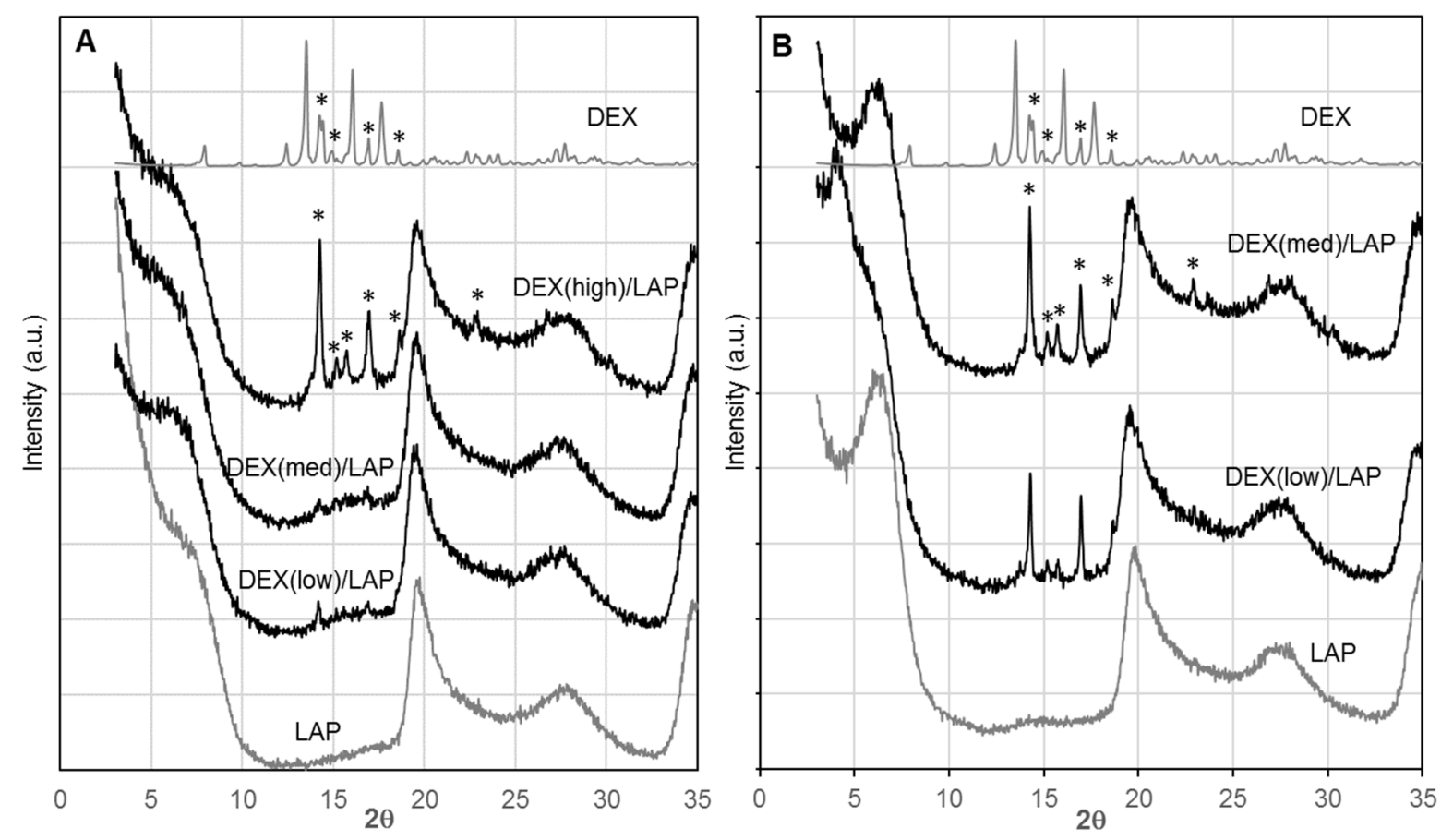

Figure 1. X-ray diffraction patterns of DEX/LAP. A) Non-oriented samples, B) Samples oriented by addition of acetone. Pattern for pure DEX is included. (*) Diffraction lines for orthorhombic DEX (JCPDS database).

The IR spectrum of DEX (Figure 2A) is mostly overlapped by the bands corresponding to the laponite structure, mainly the broad and intense band due to $\mathrm{Si}-\mathrm{O}-\mathrm{Si}$ bonds $\left(1300-1000 \mathrm{~cm}^{-1}\right)$. The presence of the organic molecule in DEX(high)/LAP is detectable in the $3000-2800 \mathrm{~cm}^{-1}$ zone, corresponding to the stretching of aliphatic $\mathrm{C}-\mathrm{H}$ bonds. Only the zone of $\mathrm{C}=\mathrm{O}$ and $\mathrm{C}=\mathrm{C}$ stretching (Figure 2B) is nearly free from laponite bands, with the only presence of the bending band of adsorbed water at $1640 \mathrm{~cm}^{-1}$. The bands at 1704 and $1662 \mathrm{~cm}^{-1}$ are assigned to the C20 (aliphatic) and C3 (unsaturated) carbonyl groups respectively (see Figure 3 for structure and numbering of DEX), whereas the $\mathrm{C}=\mathrm{C}$ bonds (C1-C2 and $\mathrm{C} 4-\mathrm{C} 5)$ appear at 1618 and $1603 \mathrm{~cm}^{-1}$. In order to detect more clearly those bands in DEX(high)/LAP, the spectrum of LAP was subtracted from the spectra of the samples (Figure $2 \mathrm{~B}$ ). The same $\mathrm{C}=\mathrm{O}$ and $\mathrm{C}=\mathrm{C}$ bands, slightly enlarged but at the same positions, are observable in DEX(high)/LAP samples. Thus, the possible interactions between DEX and LAP surface are hardly detectable by IR. 

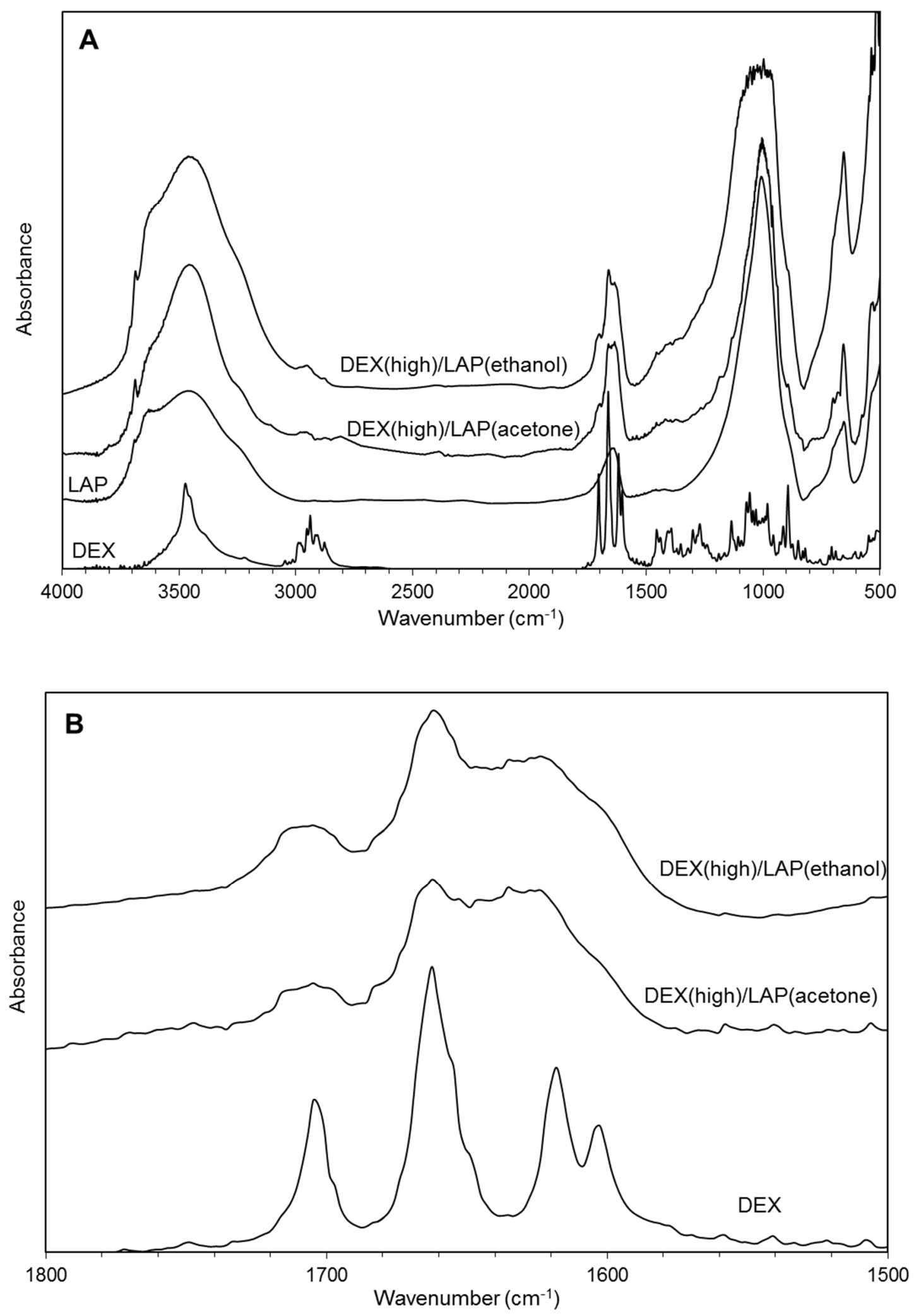

Figure 2. IR spectra of pure DEX, pristine LAP, and two samples of DEX/LAP (high content) prepared from ethanol and acetone solutions. A) $4000-500 \mathrm{~cm}^{-1}$ range. B) Enlargement of the $1800-1500 \mathrm{~cm}^{-1}$ zone. The spectrum of LAP has been subtracted to those of DEX(high)/LAP. 


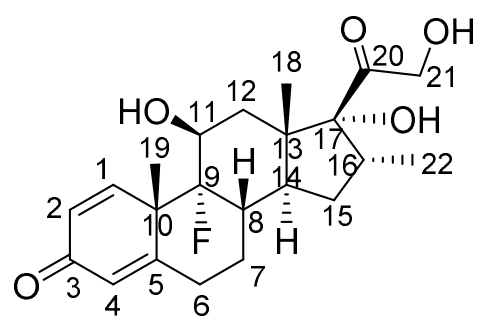

Figure 3. Molecular structure and numbering of dexamethasone (DEX).

Then the nature of the interaction between DEX and LAP surface was studied by solid state NMR, and the spectra were compared with those of DEX in solution with different solvents, namely $\mathrm{CD}_{2} \mathrm{Cl}_{2}$ in high dilution to ensure the lack of any intermolecular hydrogen bond, acetone- $d_{6}$ and dmso- $d_{6}$, as hydrogen bond acceptor (HBA) solvents with different basicity, and $\mathrm{CD}_{3} \mathrm{OD}$ as a solvent with both hydrogen bond donor (HBD) and HBA capacity (all the spectra in liquid phase can be found in Figures S1-S3 in the Supplementary Material). Assignment of the ${ }^{1} \mathrm{H}$ and ${ }^{13} \mathrm{C}$ signals was done according to APT and HSQC experiments in solution, in good agreement with the results reported in the literature for the ${ }^{1} \mathrm{H}-\mathrm{NMR}$ spectrum in dmso- $d_{6}$ [29]. Hydroxyls in positions 11,17 and 21 of dexamethasone were involved in intermolecular hydrogen bonds with the solvent in acetone and dmso, as demonstrated by the downfield shift in ${ }^{1} \mathrm{H}$ spectrum, and the shift was higher in dmso (Figure S4), according to the higher HBA character of the solvent ( $\beta=0.76 v s 0.48$ of acetone [30]). The similar chemical shift of $\mathrm{CH}-\mathrm{OH}$ and $\mathrm{CH}-\mathrm{OH}$ in both acetone and methanol indicates a similar behavior of the hydroxyls of dexamethasone in both solvents, in quite good agreement with their more similar $\beta$ values $(0.66$ and 0.48$)$. In the case of carbonyl groups, the chemical shift of $\mathrm{C} 20$ in the ${ }^{13} \mathrm{C}$ spectrum is almost not affected by the solvent, probably by the participation of an intramolecular hydrogen bond with $\mathrm{OH}$ in position 21 . On the contrary, carbonyl $\mathrm{C} 3$, as well as the conjugated $\mathrm{sp}^{2}$ carbons $\mathrm{C} 5$ and $\mathrm{C} 1$ suffer a significant downfield shift in methanol (Figure S5) [31], according to the decrease in electronic density when accepting a hydrogen (deuterium) bond.

In the case of the solid state ${ }^{13} \mathrm{C}$ CP-MAS NMR spectrum of DEX, the signals were assigned (Figure 4) according to the dipolar dephasing (DD) experiment (Figure S6) combined with the assignment from the spectra in solution. In Table 1 the chemical shifts of the spectra in solid state and in $\mathrm{CD}_{3} \mathrm{OD}$ solution are compared with that of DEX in acetone- $d_{6}$. Regarding carbonyl groups, the most significant change is observed for $\mathrm{C} 3$ and the corresponding 
conjugated carbons $\mathrm{C} 1$ and $\mathrm{C} 5$, which are shifted downfield (1.8 to $4.4 \mathrm{ppm}$ ), in a similar way as it happens in $\mathrm{CD}_{3} \mathrm{OD}$ (3.0 to $4.1 \mathrm{ppm}$ ). The donation of a hydrogen bond from the solvent can explain this effect, which is mimicked by an intermolecular hydrogen bond between the $\mathrm{OH}$ in $\mathrm{C} 17$ and the carbonyl $\mathrm{C} 3$, as shown in the X-ray structure of DEX monocrystals (Figure S7) [32]. However, in the solid state spectrum C2 and C4 are also shifted downfield, in contrast with the behavior in methanol solution, which can be attributed to the close packing in the solid state and the intermolecular influence of the $\pi$ cloud. This same effect might explain the downfield shift of methyl groups, mainly C19, and the upfield shift of methylene ones, mainly $\mathrm{C} 7$, although conformational changes from liquid to solid phase might also be responsible for this effect. Shifts in the signals within the range of 35-45 ppm are also present, but the assignment of the signals is less accurate.

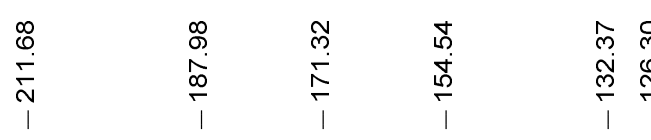

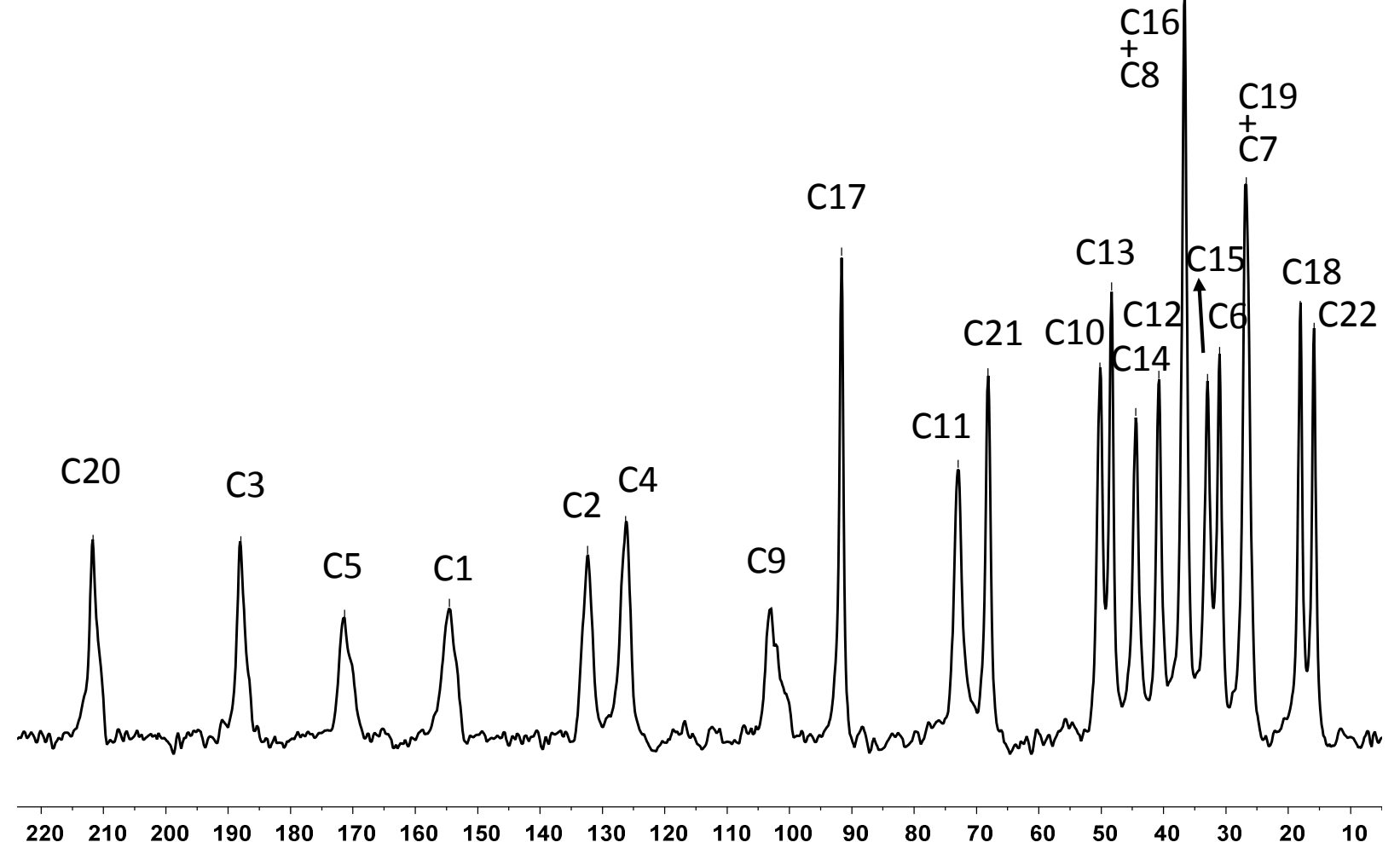

Figure 4. ${ }^{13} \mathrm{C}$ CP-MAS NMR spectrum of pure dexamethasone with the assignment of signals. 
Table 1. Comparison of $\mathrm{DEX}{ }^{13} \mathrm{C}$ chemical shifts in solution and in solid phase NMR.

\begin{tabular}{|c|c|c|c|c|}
\hline & $\delta(\mathrm{ppm})^{\mathrm{a}}$ & & & \\
\hline Carbon atom & Acetone- $d_{6}$ & Solid state & $\mathrm{CD}_{3} \mathrm{OD}$ & DEX/LAP \\
\hline $20(\mathrm{C}=\mathrm{O})$ & 212.3 & $211.7(-0.6)$ & $212.7(+0.4)$ & $212.0(-0.3)$ \\
\hline $3(\mathrm{C}=\mathrm{O})$ & 186.0 & $188.0(+2.0)$ & $189.0(+3.0)$ & $188.3(+2.3)$ \\
\hline $5(\mathrm{C}=)$ & 166.9 & $171.3(+4.4)$ & $171.0(+4.1)$ & $169.7(+2.8)$ \\
\hline $1(\mathrm{HC}=)$ & 152.7 & $154.5(+1.8)$ & $155.9(+3.2)$ & $\begin{array}{l}158.0(+5.3) \\
155.3(+2.6)^{b}\end{array}$ \\
\hline $2(\mathrm{HC}=)$ & 130.2 & $132.4(+2.4)$ & $129.8(-0.4)$ & $\begin{array}{l}130.8(+0.6) \\
128.4(-1.8)^{b}\end{array}$ \\
\hline $4(\mathrm{HC}=)$ & 125.4 & $126.3(+0.9)$ & $125.1(-0.3)$ & $126.1(+0.7)$ \\
\hline $9(\mathrm{CF})$ & 101.6 & $102.8(+1.2)$ & n.o. & $102.7(+1.1)$ \\
\hline $17(\mathrm{C}-\mathrm{OH})$ & 91.5 & 91.7 & $92.0(+0.5)$ & 91.7 \\
\hline $11(\mathrm{CH}-\mathrm{OH})$ & 72.5 & $72.9(+0.4)$ & $72.9(+0.4)$ & $71.3(-1.2)$ \\
\hline $21\left(\mathrm{CH}_{2}-\mathrm{OH}\right)$ & 67.9 & $68.1(+0.2)$ & $68.1(+0.2)$ & $68.9(+1.0)$ \\
\hline $10(\mathrm{C})$ & $\approx 49^{\mathrm{c}}$ & 50.2 & 50.2 & 49.3 \\
\hline $13(\mathrm{C})$ & & 48.3 & 49.5 & 49.3 \\
\hline $14(\mathrm{CH})$ & 44.7 & $44.5(-0.2)$ & $45.0(+0.3)$ & $44.4(-0.3)$ \\
\hline $12\left(\mathrm{CH}_{2}\right)$ & 37.1 & $40.7^{\mathrm{d}}$ & $37.5(+0.4)$ & $38.8(+1.7)$ \\
\hline $16(\mathrm{CH})$ & 36.7 & $36.7^{\mathrm{d}}$ & $37.1(+0.4)$ & $36.3(-0.4)$ \\
\hline $8(\mathrm{CH})$ & 35.1 & $36.7^{\mathrm{d}}$ & $35.6(+0.5)$ & $32.9(-2.2)$ \\
\hline $15\left(\mathrm{CH}_{2}\right)$ & 33.1 & 33.0 & $33.5(+0.4)$ & $28.1(-5.0)$ \\
\hline
\end{tabular}




\begin{tabular}{lllll}
\hline $6\left(\mathrm{CH}_{2}\right)$ & 31.6 & $31.0(-0.6)$ & $32.2(+0.6)$ & $27.7(-3.9)$ \\
$7\left(\mathrm{CH}_{2}\right)$ & 28.4 & $26.7(-1.7)$ & $28.8(+0.4)$ & $25.4(-3.0)$ \\
$19\left(\mathrm{CH}_{3}\right)$ & 23.7 & $26.7(+3.0)$ & 23.6 & $25.4(+1.7)$ \\
$18\left(\mathrm{CH}_{3}\right)$ & 17.4 & $18.1(+0.7)$ & 17.5 & $18.6(+1.2)$ \\
$22\left(\mathrm{CH}_{3}\right)$ & 15.3 & $15.9(+0.6)$ & 15.3 & $16.7(+1.4)$ \\
\hline
\end{tabular}

${ }^{\mathrm{a}}$ In parenthesis the difference with respect to the chemical shift in acetone solution $(\Delta \delta){ }^{\mathrm{b}}$ Split signal. ${ }^{\mathrm{c}}$ The signal for $\mathrm{C} 10$ is a doublet overlapped with the signal for $\mathrm{C} 13 .{ }^{\mathrm{d}}$ Tentative assignment.

The signals in the ${ }^{13} \mathrm{C}$ CP-MAS NMR spectrum of DEX(high)/LAP (Figure 5) were also assigned according with the DD spectrum (Figure S8). The chemical shifts are gathered in Table 1 and it can be seen that they are significantly different from those of pure DEX. A set of signals $(\mathrm{C} 15, \mathrm{C} 6, \mathrm{C} 7, \mathrm{C} 8)$ suffer a significant upfield shift (2.2 to $5.0 \mathrm{ppm})$ with respect to solution NMR that was not observed in the spectrum of pure DEX. On the other hand, although the intermolecular DEX-DEX interactions should ideally disappear, the effect on $\mathrm{C} 1, \mathrm{C} 3$, C5 remains (downfield shifts 2.3 to $5.3 \mathrm{ppm}$ ), as well as on C12 and C19 (1.7 ppm downfield shift), whereas the effects on the rest of carbons are of lower magnitude. This seems to indicate some polarization of the molecule (Figure S9), that can be explained by an interaction of carbonyl $\mathrm{C} 3$ and probably $\mathrm{OH}$ in $\mathrm{C} 11$ with the solid, through either coordination to $\mathrm{Na}$ ions or hydrogen bonds (hydrated $\mathrm{Na}$ ions as $\mathrm{HBD}$ and oxygen atoms in laponite sheets as HBA), that produces a deshielding of the carbon atoms involved in the interactions or in the neighborhood of them, whereas the carbon atoms in the other side of the molecule suffer an opposite shielding effect. In some cases ( $\mathrm{C} 1$ and $\mathrm{C} 2)$ the signals appear split in two, probably because of the existence of several adsorption modes on the surface. It is possible that some DEX molecules are directly involved in an interaction with the LAP surface, whereas others are supported by means of an intermolecular DEX-DEX interaction, in agreement with the presence of small DEX crystallites on LAP, as shown in the XRD patterns (Figure 1). 


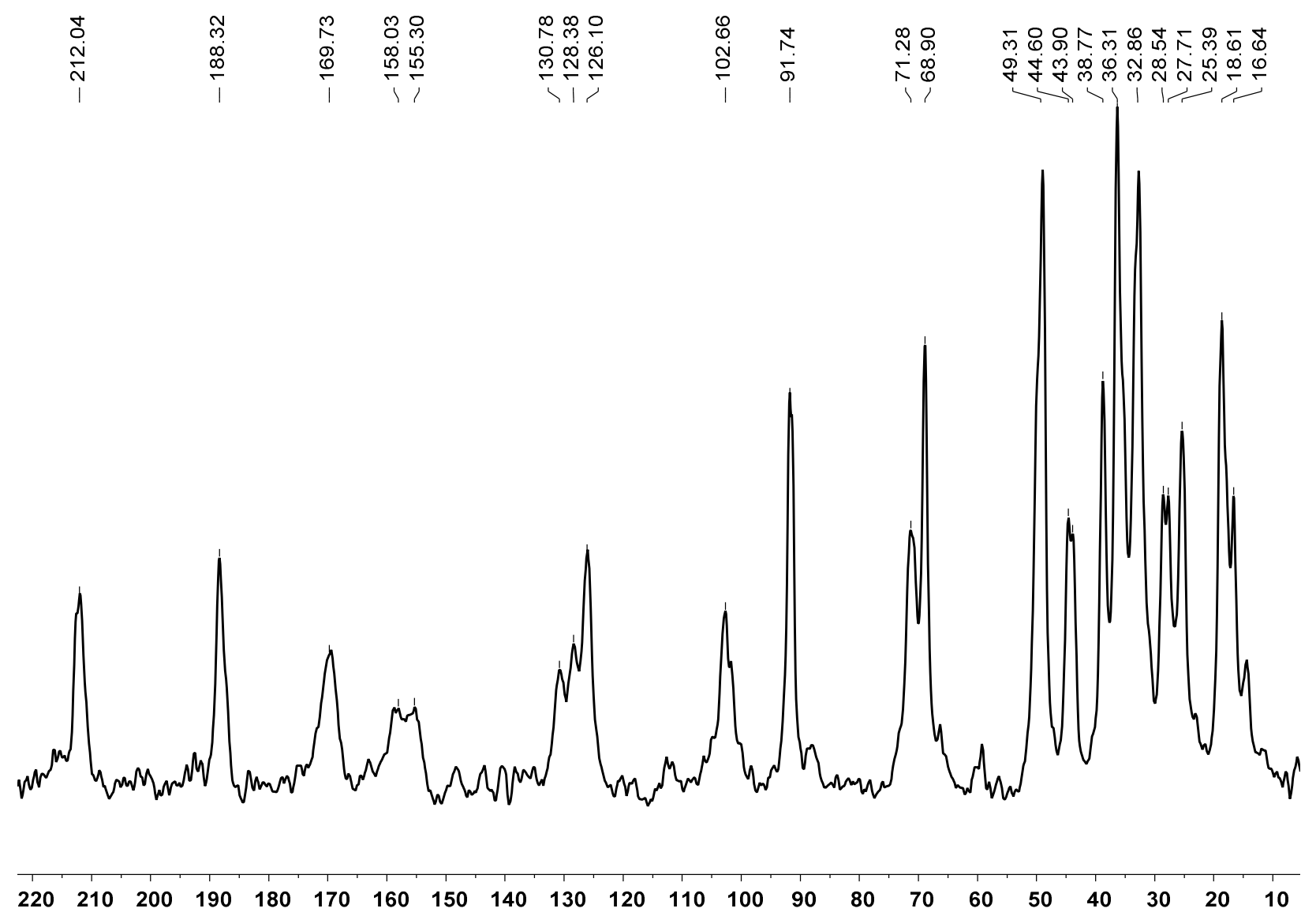

Figure 5. ${ }^{13} \mathrm{C}$ CP-MAS NMR spectrum of DEX(high)/LAP.

\subsection{In vitro $D E X$ release from $L A P$}

Vitreous humor is made up of $99 \%$ water with a saline content of about $0.9 \%$ [33], thus saline solution was chosen as a first approach to a vitreous model. As vitreous volume in rabbit is around 1.4-1.7 mL [34], the experiments of DEX delivery were carried out in $2 \mathrm{~mL}$ of saline solution by suspending $20 \mathrm{mg}$ of solid. In this way the introduced amounts of DEX, 0.95, 1.82 and $3.33 \mathrm{mg}$ for low, medium and high content DEX/LAP respectively, were by far superior to the toxic dose in rabbits $(0.4 \mathrm{mg}$ [35]), just to test if the amount present in solution reached that limit value. In this case the release mechanism should be the quite fast equilibrium between the physisorbed and the dissolved DEX, with further release once the DEX in solution is consumed. In a first set of experiments with DEX(high)/LAP and $\operatorname{DEX}($ low $) / \mathrm{LAP}$, after equilibration for 24 or $48 \mathrm{~h}$ under stirring, the solid was separated by ultracentrifugation (14000 rpm, $20 \mathrm{~min}$ ), the supernatant was analyzed by HPLC and the solid was suspended again in a new volume of saline solution to simulate the consumption of 
dissolved DEX in the vitreous humor in the eye. The average of the amounts release at the two equilibration times, with the error bars, is represented in Figure 6. As can be seen, the amounts released lead to concentrations below the reported solubility of dexamethasone in water $(0.08-0.1 \mathrm{mg} / \mathrm{mL})$. This result demonstrates the reproducibility of the in vitro measurements, as well as the role of laponite in the release control, in spite of the lack of any strong electrostatic interaction. This result contrasts with other described methods, using microparticles to encapsulate dexamethasone [36], in which DEX is immediately released according to its solubility in the extraction medium. Only in the case of the high loading sample, the obtained concentration (up to $0.07 \mathrm{mg} / \mathrm{mL}$ ) is close to the DEX solubility. These experiments do not fulfil the sink conditions, that prevent reaching concentrations too close to the solubility limit. However, those conditions are used to prevent the effect of the concentration to the release rate in kinetic release experiments. In our case, we are dealing with thermodynamic experiments, as much close as possible to the real situation, in which it would be desirable the presence of a large amount of drug, with a limited amount of liquid phase (vitreous humor), to minimize the number of intraocular injections.

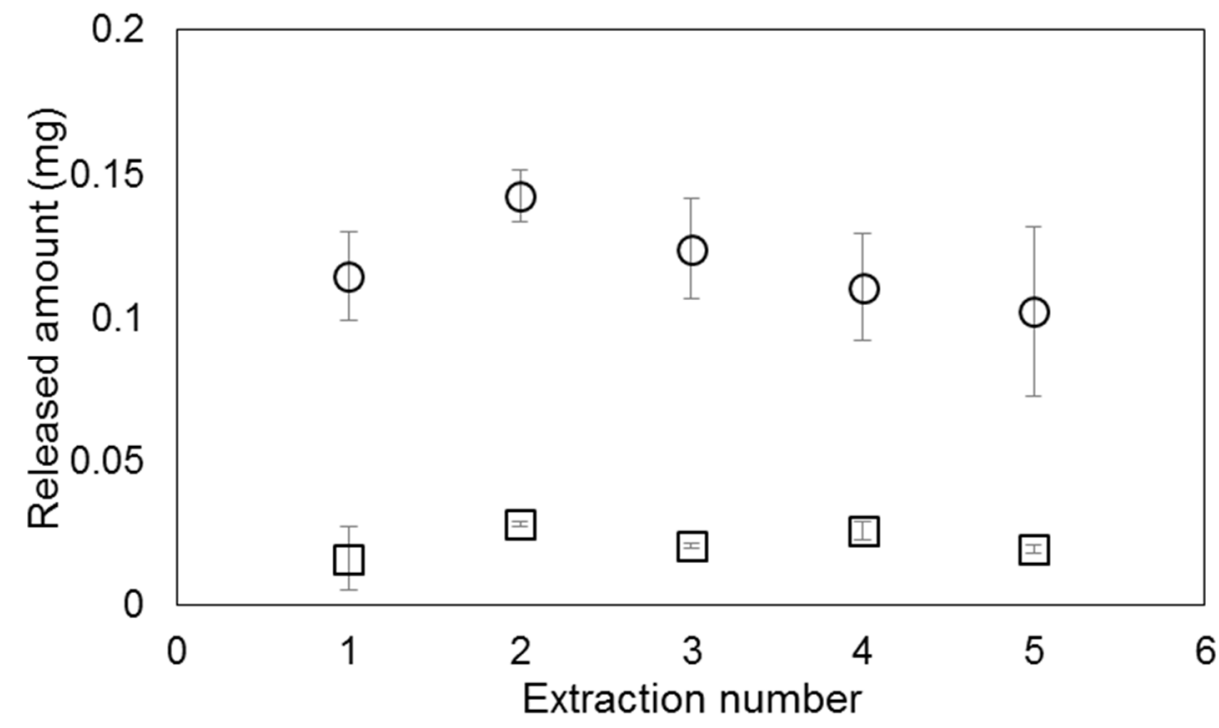

Figure 6. Release of DEX (in mg) from DEX(high)/LAP (circles) and DEX(low)/LAP (squares) to saline solution. Each point is the average of the amount released after $24 \mathrm{~h}$ and $48 \mathrm{~h}$ equilibration time. 
The cumulative DEX release in 10 consecutive saline solutions after $24 \mathrm{~h}$ equilibration time is represented in Figure 7. As can be seen, the cumulative release was almost linear, irrespective from the DEX loading on LAP, with released amounts of 0.131, 0.057 and 0.019 $\mathrm{mg}$ in average per extraction (in $2 \mathrm{~mL}$ of saline solution) for high (1:5), medium (1:10) and low $(1: 20)$ loading levels respectively. These values correspond to released percentages of around 38, 28 and 19\% of the initial DEX amount after 10 extractions for high (1:5), medium (1:10) and low (1:20) loading levels respectively. As can be seen, the percentage released also increases when the initial DEX content is higher. This behavior may indicate the existence of different interactions: a strong interaction with the clay, dominant in the sample with the lowest DEX content, and a weaker interaction, probably between DEX molecules, accounting for the higher percentage released in the samples with higher DEX content. This result is in agreement with the presence of DEX particles, detected by XRD in DEX(high)/LAP (Figure 1).

Given the role of LAP demonstrated in the first set of results of extractions in saline, it was decided to test a liquid medium more similar to the vitreous humor. Taking into account that the characteristic viscosity of vitreous is attributed to the presence of hyaluronic acid and collagen [37], we tried to mimic its physical properties by using sodium hyaluronate in saline solution. As the vitreous viscosity is in the range $300-2000 \mathrm{cP}$, it is necessary to use a concentration of at least $0.5 \%$ of sodium hyaluronate in saline solution to reach a viscosity that falls into this range [38]. Hence, the solid sample with medium loading (DEX/LAP = $1: 10 \mathrm{w} / \mathrm{w}$ ) was successively treated with $2 \mathrm{~g}$ of solution of hyaluronic acid in saline solution (1:5 w/w ratio). After $24 \mathrm{~h}$ equilibration time, the laponite was separated by ultracentrifugation and the liquid was analyzed by HPLC. The amount released to this new liquid medium (Figure 8) was lower than in the case of saline solution, with an average of $0.036 \mathrm{mg}$ per extraction (vs $0.057 \mathrm{mg}$ in saline) accounting for $1.9 \%$ of the initial amount of DEX released (vs 3.1\% in saline). Moreover, desorption to the model of vitreous humor starts to deviate from linearity earlier, confirming the slower release of dexamethasone to this medium. Again, this behavior points to the existence of two different interactions. In the first extractions, molecules with a weak interaction, probably DEX-DEX interactions instead of DEX-LAP ones, are preferably released, whereas the molecules interacting with the surface start to desorb from the fifth extraction. Thus the thermodynamic equilibrium between the DEX in solution and adsorbed onto LAP depends on several factors, including the loading of DEX on the solid and the liquid extraction medium. 

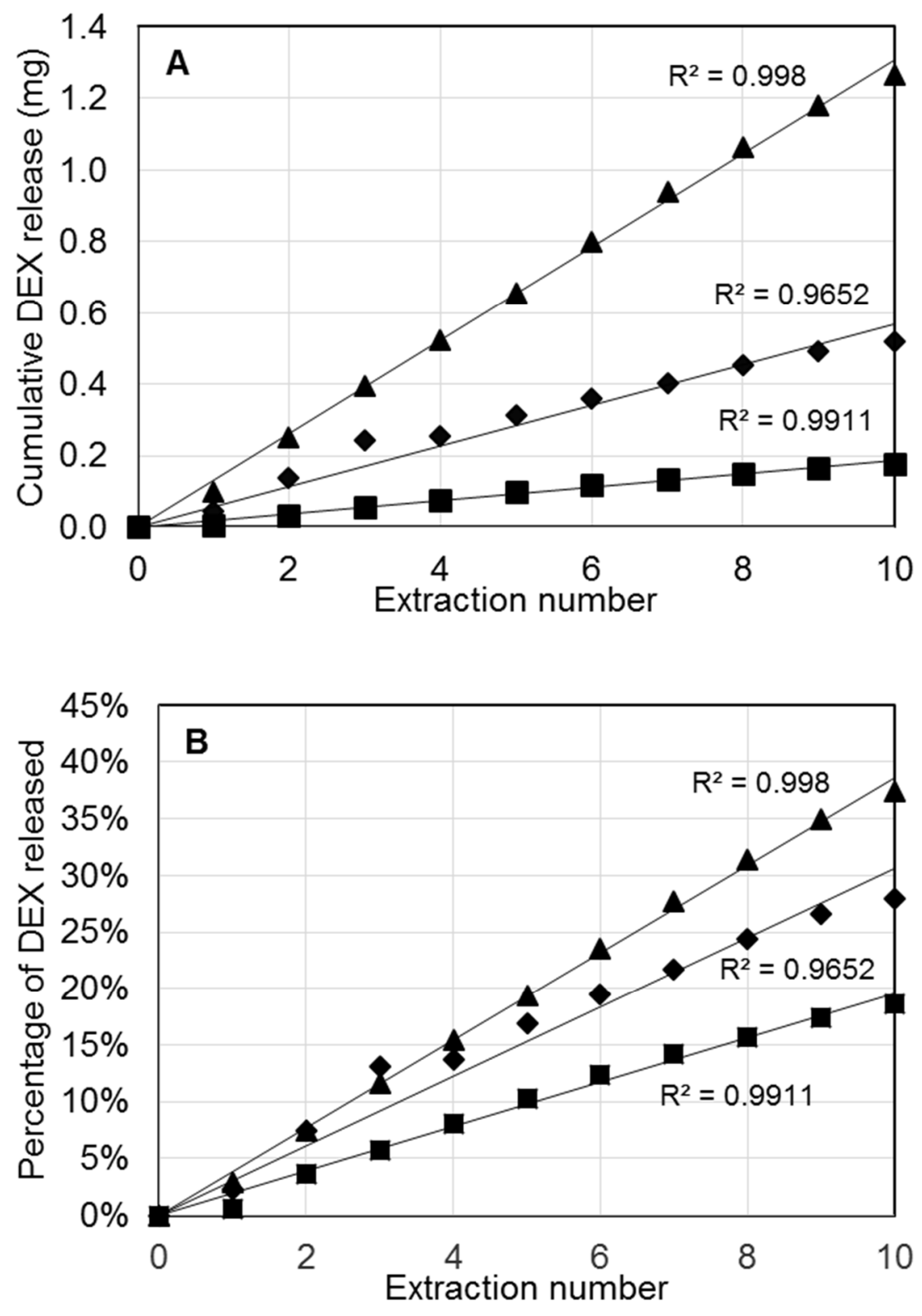

Figure 7. Cumulative release of $\operatorname{DEX}$ from $\operatorname{DEX}($ high)/LAP $(\boldsymbol{\Delta}), \operatorname{DEX}($ med $) / \operatorname{LAP}(\diamond)$, and DEX(low)/LAP ( $\square$ ) to saline solution: in mg (A) and percentage of the starting DEX content in the solid (B). 

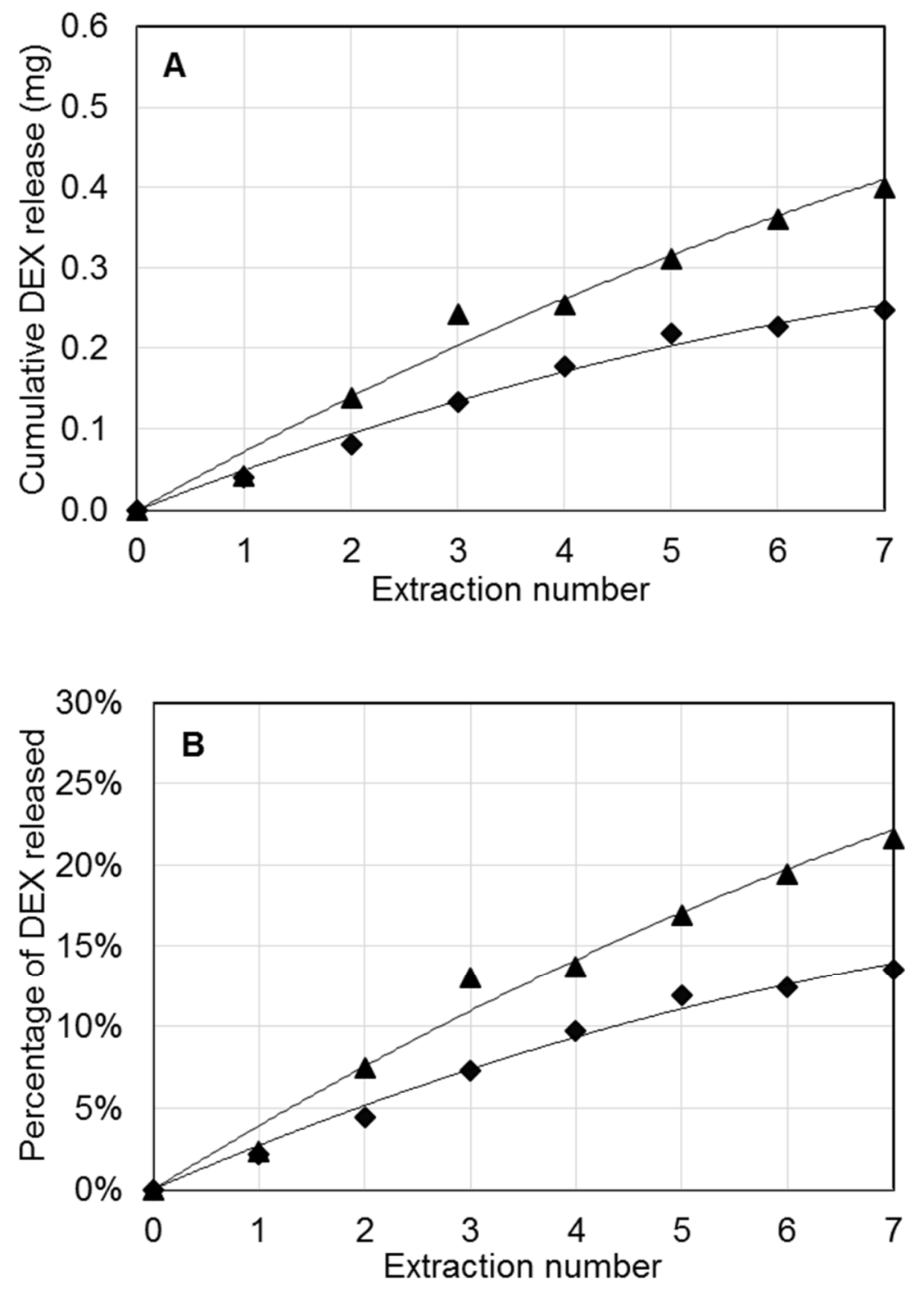

Figure 8. Cumulative release of DEX from DEX(med)/LAP to saline solution $(\boldsymbol{\Delta})$ and the model for vitreous humor $(\diamond)$ : in $\mathrm{mg}(\mathrm{A})$ and percentage of the starting DEX content in the solid (B).

Given the probable role of hydrogen bonding in the retention mechanism of DEX on LAP, the solvent used for DEX deposition (ethanol) could compete with the drug and reduce the DEX-LAP interaction. To check this possibility, the adsorption solvent was changed to acetone in an attempt to enhance the interaction of DEX with the surface. The same three loadings were tested in the release to saline solution and in Figure 9 the results are compared with those obtained with the solids prepared in ethanol. 
As can be seen, the desorbed amount is always lower than in the case of ethanol, with an earlier deviation from the linearity, showing that the interaction between dexamethasone and the laponite surface is slightly stronger, and the release is slower. However, the CP-MAS NMR spectrum of DEX/LAP prepared from acetone solution did not reveal any significant difference in chemical shifts. Only slightly broader signals were obtained, a fact that can be explained by a lower mobility of the molecules on the solid or by the existence of a larger number of different adsorption modes.

\section{Conclusion}

In this work we have demonstrated that non-ionic drugs, such as dexamethasone, can be retained on laponite, mainly through hydrogen bonding involving hydroxyl and carbonyl groups. The drug is slowly released to saline solutions, as models for vitreous humor, and the released amount depends on the loading of the drug in the solid, the composition of the liquid phase (saline solution or hyaluronic acid solution) and even the solvent used in the preparation of the solid. The transparency of the laponite support and their gels in water, as reported in the literature [21, 39] and also checked in our laboratory (Figure S10), makes it suitable for biocompatibility tests in intraocular injections. A deep study in rabbits is currently being finished, and its positive results prompted us to use this simple methodology for in vivo experiments of dexamethasone release that are currently in progress. These results also open the door for the use of laponite as a carrier for controlled delivery of other noncationic drugs bearing hydrogen bond donor groups, such as hydroxyls or carboxylic acids. 

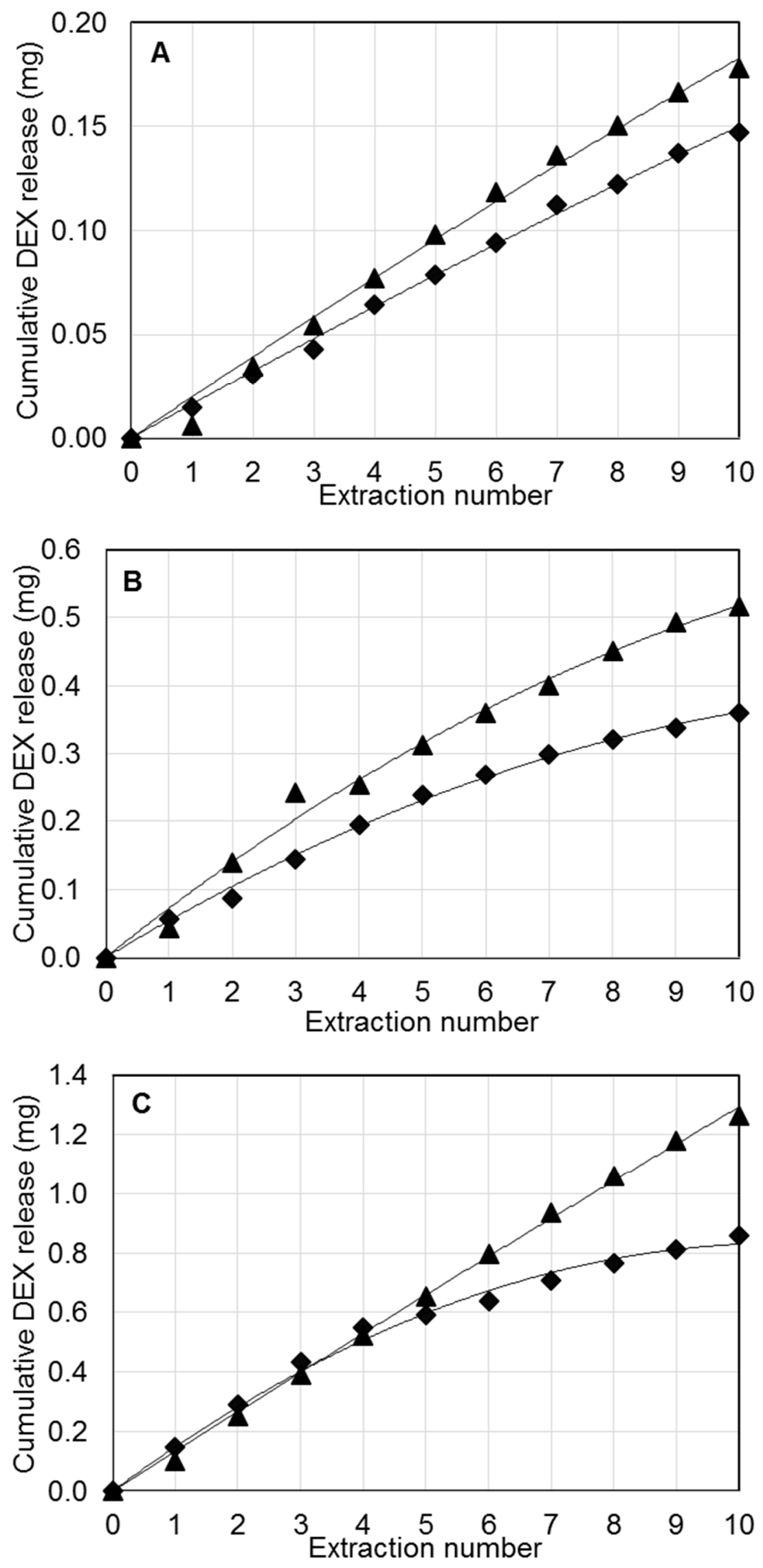
Figure 9. Cumulative release of DEX (in $\mathrm{mg}$ ) to saline solution from DEX/LAP samples prepared in ethanol $(\boldsymbol{\Delta})$ or acetone $(\diamond)$ : A) DEX(low)/LAP, B) DEX(med)/LAP, C) $\operatorname{DEX}($ high $) /$ LAP.

\section{Acknowledgements}

This study was supported by the Instituto de Salud Carlos III (project PI12/02285) and authors would like to acknowledge the financial support received from Diputación General de Aragón (E11 Group co-financed by the European Regional Development Funds).

Supplementary material. Solution NMR spectra of dexamethasone in different deuterated solvents with the assignment of signals. ${ }^{13} \mathrm{C}$ DD CP-MAS NMR spectra of dexamethasone and DEX/LAP. Hydrogen bonds present in the solid dexamethasone from its X-ray structure. Transparency test of a laponite dispersion in saline solution.

\section{References}

[1] Fagan, X. J.; Al-Qureshi, S. Intravitreal injections: a review of the evidence for best practice, Clin. Exp. Ophthalmol. 2013, 41, 500-507.

[2] Velez, G.; Whitcup, S. M. New developments in sustained release drug delivery for the treatment of intraocular disease, Br. J. Ophthalmol. 1999, 83, 1225-1229.

[3] Jonas, J. B.; Spandau, U. H.; Schlichtenbrede, F. Short-term complications of intravitreal injections of triamcinolone and bevacizumab, Eye (London) 2008, 22, 590-591.

[4] Yasukawa, T.; Ogura, Y.; Sakurai, E.; Tabata, Y.; Kimura, H. Intraocular sustained drug delivery using implantable polymeric devices, Adv. Drug Delivery Rev. 2005, 57, 2033-2046.

[5] Kompella, U. B.; Amrite, A. C.; Pacha Ravi, R.; Durazo, S.A. Nanomedicines for back of the eye drug delivery, gene delivery, and imaging, Prog. Retin. Eye Res. 2013, 36, 172-198. 
[6] Yasin, M. N.; Svirskis, D.; Seyfoddin, A.; Rupenthal, I. D. Implants for drug delivery to the posterior segment of the eye: a focus on stimuli-responsive and tunable release systems, $J$. Control. Release 2014, 196, 208-221.

[7] Zignani, M.; Einmahl, S.; Baeyens, V.; Varesio, E.; Veuthey, J. L.; Anderson, J.; Heller, J.; Tabatabay, C.; Gurny, R. A poly(ortho ester) designed for combined ocular delivery of dexamethasone sodium phosphate and 5-fluorouracil: subconjunctival tolerance and in vitro release, Eur. J. Pharm. Biopharm. 2000, 50, 251-255.

[8] Fialho, S. L.; Behar-Cohen, F.; Silva-Cunha, A. Dexamethasone-loaded poly( $\varepsilon-$ caprolactone) intravitreal implants: A pilot study, Eur. J. Pharm. Biopharm. 2008, 68, 637646.

[9] Haghjou, N.; Soheilian, M.; Abdekhodaie, M. J. Sustained release intraocular drug delivery devices for treatment of uveitis, J. Ophthalmic Vis. Res. 2011, 6, 317-329.

[10] Rodríguez-Agirretxe, I.; Vega, S. C.; Rezola, R.; Vecino, E.; Mendicute, J.; SuarezCortes, T.; Acera, A. The PLGA implant as an antimitotic delivery system after experimental trabeculectomy, Invest. Ophthalmol. Vis. Sci. 2013, 54, 5227-5235.

[11] Zhang, L.; Shen, W.; Luan, J.; Yang, D.; Wei, G.; Yu, L.; Lu, W.; Ding, J. Sustained intravitreal delivery of dexamethasone using an injectable and biodegradable thermogel, Acta Biomateralia 2015, 23, 271-281.

[12] Mishra, G. P.; Kinser, R.; Wierzbicki, I. H.; Alany, R. G.; Alani, A. W. G. In situ gelling polyvalerolactone-based thermosensitive hydrogel for sustained drug delivery, Eur. J. Pharm. Biopharm. 2014, 88, 397-405.

[13] Ito, T.; Fraser, I. P.; Yeo, Y.; Highley, C. B.; Bellas, E.; Kohane, D. S. Antiinflammatory function of an in situ cross-linkable conjugate hydrogel of hyaluronic acid and dexamethasone, Biomaterials 2007, 28, 1778-1786.

[14] Xiao, L.; Tong, Z.; Chen, Y.; Pochan, D. J.; Sabanayagam, C. R.; Jia, X. Hyaluronic acid-based hydrogels containing covalently integrated drug depots: implication for controlling inflammation in mechanically stressed tissues, Biomacromolecules 2013, 14, $3808-3819$. 
[15] Viseras, C.; Aguzzi, C.; Cerezo, P.; López-Galindo, A. Uses of clay minerals in semisolid health care and therapeutic products, Appl. Clay Sci. 2007, 36, 37-50.

[16] Ghadiri, M.; Chrzanowski, W.; Rohanizadeh, R. Biomedical applications of cationic clay minerals, $R S C A d v$. 2015, 5, 29467-29481.

[17] Aguzzi, C.; Cerezo, P.; Viseras, C.; Caramella, C. Use of clays as drug delivery systems: possibilities and limitations, Appl. Clay Sci. 2007, 36, 22-36.

[18] Viseras, C.; Cerezo, P.; Sánchez, R.; Salcedo, I.; Aguzzi, C. Current challenges in clay minerals for drug delivery, Appl. Clay Sci. 2010, 48, 291-295.

[19] de Sousa Rodrigues, L. A.; Figueiras, A.; Veiga, F.; de Freitas, R. M.; Cunha Nunes, L. C.; da Silva Filho, E. C.; da Silva Leite, C. M. The systems containing clays and clay minerals for modified drug release: a review, Colloids Surf. B 2013, 103, 642-651.

[20] Rodrigues da Silva, G.; da Silva-Cunha Jr, A.; Behar-Cohen, F.; Ayres, E., Oréfice, R. L. Biodegradable polyurethane nanocomposites containing dexamethasone for ocular route, Mater. Sci. Eng. C 2011, 31, 414-422.

[21] Ruzicka, B.; Zaccarelli, E. A fresh look at the laponite phase diagram, Soft Matter 2011, 7, 1268-1286.

[22] Jung, H.; Kim, H.-M.; Choy, Y. B.; Hwang, S.-J.; Choy, J.-H. Laponite-based nanohybrid for enhanced solubility and controlled release of itraconazole, Int. J. Pharm. 2008, 349, 283-290.

[23] Park, J. K.; Choy, Y. B.; Oh, J.-M.; Kim, J. Y.; Hwang, S.-J.; Choy, J.-H. Controlled release of donepezil intercalated in smectite clays, Int. J. Pharm. 2008, 359, 198-204.

[24] Ghadiri, M.; Hau, H.; Chrzanowski, W.; Agus, H.; Rohanizadeh, R. Laponite clay as a carrier for in situ delivery of tetracycline, RSC Adv. 2013, 3, 20193-20201.

[25] Wang, S.; Wu, Y.; Guo, R.; Huang, Y.; Wen, S.; Shen, M.; Wang, J.; Shi, X. Laponite nanodisks as an efficient platform for doxorubicin delivery to cancer cells, Langmuir 2013, $29,5030-5036$. 
[26] Li, K.; Wang, S.; Wen, S.; Tang, Y.; Li, J.; Shi, X.; Zhao, Q. Enhanced in vivo antitumor efficacy of doxorubicin encapsulated within laponite nanodisks, ACS Appl. Mater. Interf. 2014, 6, 12328-12334.

[27] Wu, Y.; Guo, R.; Wen, S.; Shen, M.; Zhu, M.; Wang, J.; Shi, X. Folic acid-modified laponite nanodisks for targeted anticancer drug delivery, J. Mater. Chem. B 2014, 2, 74107418 .

[28] Volkov, A. G.; Paula, S.; Deamer, D. W. Two mechanisms of permeation of small neutral molecules and hydrated ions across phospholipid bilayers, Bioelectrochem. Bioenerg. 1997, 42, 153-160.

[29] Liu, M.; Farrant, R. D.; Nicholson, J. K.; Lindon, J. C. Selective detection of ${ }^{1}$ H NMR resonances of $\mathrm{CH}_{\mathrm{n}}$ groups using a heteronuclear maximum-quantum filter and pulsed field gradients, J. Magn. Reson. B 1995, 106, 270-278.

[30] Reichardt, C. Solvents and solvent effects in organic chemistry, 3rd ed.; Wiley-VCH: Weinheim, 2003; p 433.

[31] Marr, D. H.; Stothers, J. B. ${ }^{13}$ C N.M.R. studies. Part VI. Carbon-13 spectra of $\alpha, \beta-$ unsaturated carbonyl compounds, Can. J. Chem. 1965, 43, 596-607.

[32] Raynor, J. W.; Minor, W.; Chruszcz, M. Dexamethasone at 119 K, Acta Cryst. E 2007, E63, o2791-02793.

[33] Bishop, P. N. Structural macromolecules and supramolecular organization of the vitreous gel, Prog. Retin. Eye Res. 2000, 19, 323-344.

[34] Meredith, T. A. in Intraocular drug delivery, Jaffe, G. J.; Ashton, P.; Pearson, P. A. eds, Taylor \& Francis, Boca Raton, 2006; pp 85-95.

[35] Graham, R. O.; Peyman, G. A. Arch. Ophthalmol. 1974, 92, 149-154.

[36] Gómez-Gaete, C.; Tsapis, N.; Silva, L.; Bourgaux, C.; Besnard, M.; Bochot, A.; Fattal, E. Supramolecular organization and release properties of phospholipid-hyaluronan microparticles encapsulating dexamethasone, Eur. J. Pharm. Biopharm. 2008, 70, 116-126. 
[37] Donati, S.; Caprani, S. M.; Airaghi, G.; Vinciguerra, R.; Bartalena, L.; Testa, F.;

Mariotti, C.; Porta, G.; Simonelli, F.; Azzolini, C. Vitreous substitutes: the present and the future. BioMed Res. Int. 2014, Article ID 351804, 1-12.

[38] Krause, W. E.; Bellomo, E. G.; Colby, R. H. Rheology of sodium hyaluronate under physiological conditions. Biomacromol. 2001, 2, 65-69.

[39] Le Luyer, C.; Lou, L.; Bovier, C. ; Plenet, J. C. ; Dumas, J. G. ; Mugnier, J. A thick solgel inorganic layer for optical planar waveguide applications. Opt. Mater. 2001, 18, 211-217. 